\title{
EFECTO DE LA ENDOGAMIA SOBRE PARÁMETROS PRODUCTIVOS EN VACAS HOLSTEIN Y JERSEY DE COSTA RICA
}

\author{
Judyana Aguirre-Valverde*, Bernardo Vargas-Leitón ${ }^{* *}$, Juan José Romero-Zúñigal ${ }^{1 / * * *}$ \\ Palabras clave: Consanguinidad, producción 305 días, ganado lechero, Costa Rica. \\ Keywords: Inbreeding, 305 day milk yield, dairy cattle, Costa Rica.
}

Recibido: $13 / 03 / 13$

\section{RESUMEN}

Se realizó un estudio longitudinal retrospectivo en 208148 registros de vacas Holstein y Jersey de 545 hatos lecheros especializados presentes en la Base Nacional de Datos VAMPP Bovino, del Centro Regional de Informática para la Producción Animal Sostenible (CRIPAS), de la Escuela de Medicina Veterinaria, Universidad Nacional (EMV-UNA), período 1995-2010. Se analizó el efecto de la endogamia sobre la producción corregida a 305 días (PC305d) y la vida productiva -calculada según la fórmula USDA--, con regresión lineal múltiple, mediante un modelo mixto. Se usó como punto de corte, un valor de coeficiente de endogamia (F) de $\geq 6,25 \%$ para valores altos. Además, se estimó la posibilidad de que una vaca con $\mathrm{F} \geq 6,25 \%$ presente parámetros productivos bajos, mediante regresión logística no condicional. El análisis de supervivencia, mediante la distribución de Weibull, determinó el efecto de endogamia sobre la vida productiva. Se encontró un $17,9 \%$ de animales con endogamia, siendo la raza Holstein la de más animales consanguíneos, aunque la Jersey fue la que tuvo, en promedio, los niveles más elevados. Las vacas con baja endogamia produjeron $+140,4$

\footnotetext{
1 Autor para correspondencia. Correo electrónico: juan.romero.zuniga@una.cr

* Maestría en Producción Animal Sostenible, Universidad Nacional. Heredia, Costa Rica.
}

Aceptado: 04/09/13

\begin{abstract}
Effect of inbreeding on productive parameters in Costa Rican Holstein and Jersey cows. A retrospective longitudinal study was conducted with 208148 records of Holstein and Jersey cows from 545 specialized dairy herds present in the VAMPP Bovino National Data Base, of the Regional Center of Informatics for Sustainable Animal Production (CRIPAS), School of Veterinary Medicine, National University, from 1995 to 2010. The effect of inbreeding on the 305-day-corrected milk yield (PC305d) and productive life--calculated according to the USDA formula--, was analyzed by multiple linear regression, using a mixed model. A cut-off point value of $\geq 6.25 \%$ for the coefficient of inbreeding (F) was used to delimit high $F$ levels. In addition, the possibility for a cow with $\mathrm{F} \geq 6.25 \%$ having low productive parameters was calculated by non conditional logistic regression. The survival analysis, using the Weibull distribution, was used to determine the effect of inbreeding on the productive life. A $17.9 \%$ of animals with inbreeding was found, being Holstein the race with more inbred cows, although Jersey had, on the average, the highest inbreeding levels. Cows

\footnotetext{
** Posgrado en Ciencias Veterinarias Tropicales, Universidad Nacional. Heredia, Costa Rica. Programa de Investigación en Medicina Poblacional. Escuela de Medicina Veterinaria. Universidad Nacional. Costa Rica.
} 
kg PC305d en relación con las que tienen niveles elevados $(\mathrm{p}<0,01)$. Las vacas con alta endogamia tuvieron más riesgo de PC 305d $<5000 \mathrm{~kg}$ (Jersey) $\mathrm{y}<6500 \mathrm{~kg}$ (Holstein) que las de baja endogamia (OR=1,3; IC95\%: 1,2-1,4). Se observó, para las razas Holstein y Jersey, $+10,3$ meses y $+19,3$ meses de vida productiva (USDA) para los animales con $\mathrm{F}$ bajo. Se concluye que existe un efecto negativo de la consanguinidad elevada sobre la vida productiva (USDA) y sobre la PC 305d de las vacas Holstein y Jersey en fincas lecheras de Costa Rica.

\section{INTRODUCCIÓN}

La endogamia (consanguinidad) puede definirse como el apareamiento entre animales emparentados entre sí genéticamente por ancestros comunes (Wright 1922). La endogamia disminuye heterocigosidad y aumenta la frecuencia de genes deletéreos recesivos, lo que puede reducir el rendimiento y la viabilidad fenotípica. Este fenómeno conocido como la depresión endogámica, afecta parámetros productivos y reproductivos que generalmente provoca una disminución de la rentabilidad de granjas lecheras (Weigel y Lin 2000).

Varios estudios demuestran que hay una fuerte evidencia de esta depresión endogámica, que afecta a todos los rasgos de producción $(\mathrm{kg} / 305 \mathrm{~d})$, porcentaje de grasa y proteína en leche (Cassell 1999, Maiwashe et ál. 2008), días de vida productiva (Smith et ál. 1998) y pérdida de fortaleza o capacidad de sobrevivencia (De Alba 1964, McParland et ál. 2007). Se han observado disminuciones de rendimiento productivo por cada $1 \%$ de incremento de endogamia de hasta 58,02 $\mathrm{kg}$ de leche en cada lactancia para la raza Jersey (Maiwashe et ál. 2008) y de 29,6 kg para la raza Holstein (Wiggans et ál. 1995), 358 kg (Cassell 1999) y $177 \mathrm{~kg}$ (Smith et ál. 1998) menos de producción total de leche, 13 d (Cassell 1999) menos with low F produced $+140.4 \mathrm{~kg}$ PC305d compared to those having elevated $\mathrm{F}$ levels $(\mathrm{p}<0.01)$. Cows with high F had increased risk of PC305d $<5000$ $\mathrm{kg}$ (Jersey) and $<6500 \mathrm{~kg}$ (Holstein) than those with low inbreeding coefficient $(\mathrm{OR}=1.3$; IC95\%: $1.2-1.4)$. It was observed, +10.3 months and +19.3 months of productive life (USDA) for Holstein and Jersey cows with low inbreeding coefficient. We conclude that there is a negative effect of elevated inbreeding coefficient on the productive life (USDA) and the PC305d of Holstein and Jersey cows in Costa Rican dairy farms.

de vida productiva, $10 \mathrm{~d}$ (Cassell 1999) y 4,80 d (Smith et ál. 1998) menos de producción de leche.

Las vacas inician su vida productiva en el momento en que empieza la producción de leche en su primera lactancia y sigue una tendencia al incremento hasta lograr la mayor producción en la tercera lactancia (Colina et ál. 2000). En vacas de producción láctea, el término vida útil o vida productiva es utilizado para indicar el período comprendido entre la fecha del primer parto y el día de secado o descarte del último parto, es decir, los días totales de producción de leche, más los días seca (Ducrocq y Solkner 1998, Chirinos et ál. 1999). Resultados de estudios muestran 44,4 (De Alba 1964) y 42,9 (Orrego et ál. 2003) meses de vida productiva en la raza Holstein, mientras que para la raza Jersey se reportan 55,32 $\mathrm{m}$ (De Alba 1964).

Por otro lado, para el USDA (United States Department of Agriculture) la vida productiva consiste en la asignación de créditos a una vaca por cada día de ordeño que permanece en el hato, es decir, la vaca no recibe créditos por los días secos. Estos créditos se suman a lo largo de todas las lactancias disponibles para obtener un acumulado final que se interpreta en términos meses de vida productiva (Caraviello 2004, Van Raden et ál. 2006). 
El objetivo de esta investigación es caracterizar la endogamia y determinar su efecto sobre la producción de leche corregida a 305 d y sobre la vida productiva según USDA en vacas de raza Holstein y Jersey en hatos lecheros especializados de Costa Rica.

\section{MATERIALES Y MÉTODOS}

\section{Fuente de datos}

Se realizó un estudio longitudinal retrospectivo con los registros de 1395 fincas lecheras de Costa Rica. Esta información pertenece a la base de datos nacional del programa VAMPP Bovino (Veterinary Automated Management and Production Control Programme) (Noordhuizen y Buurman 1984), en el Centro Regional de Informática para la Producción Animal Sostenible (CRIPAS), de la Escuela de Medicina Veterinaria - Universidad Nacional (EMV-UNA). De estos datos, se seleccionaron las vacas que nacieron en el periodo comprendido entre el $1^{\circ}$ de enero de 1995 y el 30 de diciembre de 2010, y que tuvieran datos de genealogía que permitieran el cálculo de su coeficiente de consanguinidad (F). En total, 545 hatos lecheros cumplieron con los criterios de inclusión del estudio.

\section{Método de recolección de datos}

Diariamente, en cada finca, los productores capturan en una libreta de apuntes los datos de los eventos que ocurren. Luego, esta información es introducida en el programa VAMPP Bovino por ellos mismos o por los técnicos que trabajan y ofrecen asistencia en la granja. El personal del proyecto CRIPAS recolecta y centraliza, al menos 2 veces al año, la información contenida en el programa VAMPP Bovino de cada fina en forma descentralizada (Romero 2005). Hoy día, VAMPP Bovino es utilizado por alrededor de 1500 granjas lecheras especializadas en Costa Rica, algunas con información de hace 30 años.

\section{Descripción de las variables}

La variable independiente principal es el coeficiente de endogamia (F) de cada vaca lechera, siendo las variables dependientes la producción de leche y vida productiva. Se tomaron en consideración otras variables y fuentes de variación como raza, número de lactancias, ecozona, hato, año y época de parto, las cuales pueden incidir en las variables dependientes, además del nivel de consanguinidad. Las variables dependientes e independientes y la forma en que se utilizaron se describen en el Cuadro 1. 
Cuadro 1. Descripción de las variables productivas utilizadas en el análisis de la situación de endogamia en fincas lecheras de Costa Rica, período 1995-2010.

\begin{tabular}{|c|c|c|c|c|}
\hline Variable & Condición & Tipo & Nivel & Descripción \\
\hline \multirow[t]{5}{*}{ Producción de leche corregida a 305 d } & Dependiente & Continua & & $\mathrm{kg}$ \\
\hline & & Discreta & Bajo & $<5000$ kg Jersey \\
\hline & & & & $<6500 \mathrm{~kg}$ Holstein \\
\hline & & & Alto & $\geq 5000 \mathrm{~kg}$ Jersey \\
\hline & & & & $\geq 6500 \mathrm{~kg}$ Holstein \\
\hline \multirow[t]{3}{*}{ Vida productiva } & Dependiente & Continua & & Valor absoluto en meses \\
\hline & & Discreta & Bajo & $<40$ meses \\
\hline & & & Alto & $\geq 40$ meses \\
\hline \multirow[t]{3}{*}{ Endogamia } & Independiente & Continua & & Porcentaje \\
\hline & & Discreta & Bajo & $<6,25 \%^{*}$ \\
\hline & & & Alto & $\geq 6,25 \%$ \\
\hline \multirow[t]{2}{*}{ Raza } & Independiente & Discreta & Holstein & $\geq 6 / 8$ Holstein \\
\hline & & & Jersey & $\geq 6 / 8$ Jersey \\
\hline \multirow[t]{3}{*}{ Días de lactancia } & Independiente & Discreta & Bajo & $<275 \mathrm{~d}$ \\
\hline & & & Normal & $275 \mathrm{~d}-325 \mathrm{~d}$ \\
\hline & & & Alto & $>325 \mathrm{~d}$ \\
\hline \multirow[t]{6}{*}{ Número de lactancia } & Independiente & Continua & & \\
\hline & & Discreta & $1^{\mathrm{a}}$ & Primerizas \\
\hline & & & $2^{\mathrm{a}}$ & Segunda \\
\hline & & & $3^{\mathrm{a}}$ & Tercera \\
\hline & & & $4^{\mathrm{a}}$ & Cuarta \\
\hline & & & $5^{\mathrm{a}}$ & $\geq$ Quinta \\
\hline Ecozonas & Independiente & Discreta & 1 a 9 & Zonas de vida** \\
\hline Hato & Independiente & Discreta & $1-1395$ & Número consecutivo \\
\hline Año de parto & Independiente & Discreta & $1995-2010$ & Período del estudio \\
\hline Hato.año-1 & Independiente & Discreta & $1995-2010$ & Base de datos VAMPP \\
\hline \multirow[t]{2}{*}{ Época de parto } & Independiente & Discreta & $0=$ Seca & Diciembre, Enero-Abril \\
\hline & & & 1= Lluviosa & Mayo-Noviembre \\
\hline
\end{tabular}

*Florio 2005.

*** Holdridge 1987.

\section{Edición y análisis de datos}

Los animales nacidos en años anteriores a 1985 o después de 2008, se tomaron como nacidos en esos años. Si el año de parto era mayor a 2010, se reubicaron en ese año; esto debido al bajo volumen de datos para fechas de nacimiento o de parto para esos años utilizados como límites. Esto se hizo con el fin de incorporar esos datos al análisis, una vez que se determinó que la cantidad era muy baja y que el efecto de hacer ese agrupamiento no afectó de manera significativa los resultados.

Para determinar el efecto de la endogamia sobre los parámetros reproductivos se incluyeron en el estudio únicamente las vacas Holstein y Jersey; asimismo, los cruces con $75 \%$ o más de la raza fueron considerados como "puras".

En cuanto a la variable zonas de vida según Holdridge (1987) se eliminaron 3 zonas por presentar pocos datos y que no aportaban mucha información al estudio: bosque muy húmedo 
montano (bmh-M), bosque pluvial premontano (bp-PM) y bosque seco tropical (bs-T), las utilizadas en este estudio se describen en el Cuadro 2.

Se utilizó la fórmula de cálculo de vida productiva según el Departamento de Agricultura de los Estados Unidos de América (USDA) (Van Raden et ál. 2006). Las vacas que tenían una vida productiva según USDA $>120 \mathrm{~m}$ se asumió como $=120$, la producción corregida a 305 d $<500 \mathrm{~kg}$ se trasladó a 500. Esto con el fin de eliminar valores

Cuadro 2. Rangos de variación en altitud, precipitación y temperatura en zonas agroecológicas de Costa Rica con presencia de sistemas de producción lecheros ${ }^{1}$.

\begin{tabular}{llccc}
\hline \multicolumn{1}{c}{ Zona ecológica } & Código & $\begin{array}{c}\text { Altitud } \\
(\mathrm{msnm})\end{array}$ & $\begin{array}{c}\text { Precipitación } \\
\left(\mathrm{mm} . \mathrm{año}^{-1}\right)\end{array}$ & Temperatura $\left({ }^{\circ} \mathrm{C}\right)$ \\
\hline B. muy húmedo tropical & bmh-T & $0-500$ & $4000-8000$ & $>24$ \\
B. muy húmedo premontano & bmh-P & $500-1500$ & $2000-4000$ & $18-24$ \\
B. húmedo montano bajo & bh-MB & $1500-2000$ & $1000-2000$ & $12-17$ \\
B. pluvial montano bajo & bp-MB & $1500-2000$ & $4000-8000$ & $12-17$ \\
B. húmedo tropical & bh-T & $0-500$ & $2000-4000$ & $>24$ \\
B. húmedo premontano & bh-P & $500-1500$ & $1000-2000$ & $18-24$ \\
B. muy húmedo montano bajo & bmh-MB & $1500-2000$ & $2000-4000$ & $12-17$ \\
\hline
\end{tabular}

${ }^{1}$ Tomado de Vargas y Ulloa (2008). Desarrollado con base en Holdridge (1987).

extremos y trabajar dentro de los rangos máximos y mínimos plausibles.

El cálculo de vida productiva está basado en el método utilizado por AIPL-USDA, según se describe en Van Raden et. ál (2006). De acuerdo con este método la vida productiva de una vaca se mide en créditos, los cuales se asignan en función de los días en ordeño que permanece en el hato. El valor del crédito por día en ordeño es variable y se obtiene mediante la siguiente fórmula:

$$
w_{i, t}=\frac{Y_{i, t}-Y_{\min }}{\bar{Y}_{4,305}-Y_{\min }}
$$

En esta fórmula se compara la producción diaria esperada de la vaca para cada día de lactancia $\left(Y_{i, t}\right)$ contra la producción promedio diaria de una lactancia base o de referencia $\left(\bar{Y}_{4,305}\right)$. La producción diaria esperada es obtenida por métodos de interpolación no lineal basados en producciones diarias observadas $\left(\mathrm{Y}_{\mathrm{i}, \mathrm{t}}\right)$ y curvas de lactancia estandarizadas generadas a nivel local. La lactancia base para cada raza dentro de la población local, correspondió a la lactancia estándar del grupo de vacas de la zona BMH-P, con nivel de producción intermedio, con parto en época lluviosa y con una edad de 4 años. Los créditos asignados a cada día de cada una de las lactancias de la vaca, son agregados en un solo estimado final y se expresan en términos de meses de vida productiva. Cabe agregar que no se otorgan créditos en días de lactancia que sobrepasen los 16 meses. Además tampoco se otorgan créditos por producciones menores a Ymin $(5 \mathrm{~kg})$ valor que fue definido con base en la producción esperada más baja obtenida en las curvas estándares locales.

\section{Procesamiento estadístico}

El procesamiento de datos se realizó mediante 3 fases. En la primera fase del análisis se hicieron estimaciones de supervivencia de vida productiva según USDA para el modelo 
paramétrico mediante la distribución de Weibull (Van Raden et ál. 2006), de la rutina PROC LIFEREG (SAS/STAT® ver 9.2).

La segunda fase fue la estimación del efecto absoluto mediante la regresión lineal múltiple y un modelo mixto (PROC MIXED, SAS/STAT ${ }^{\circledR}$ ver 9.2). Se creó un modelo para cada variable dependiente.

El modelo estadístico para producción de leche corregida a 305 d es:

$$
Y_{i j k l m n}=\mu O+e n d o_{i}+h a p_{j}+e p_{k}+r a z a l+n l a c m+e c o n+e_{i j k l m n}
$$

Donde:

$Y_{i j k l m n}=$ producción de leche $(\mathrm{kg})$

$\mu O=$ media general de la variable de respuesta

endo ${ }_{i}=$ efecto fijo de la $i$-ésima endogamia $(i=$ $0 \ldots$; porcentaje de endogamia)

$h a p_{j}=$ efecto aleatorio del j-ésimo hato/año de parto

$e p_{k}=$ efecto fijo de $k$-ésima época de parto $(k=$ $0-1 ; 0=$ seca, $1=$ lluviosa)

$r a z a_{l}=$ efecto fijo de la $l$-ésima raza $(l=1-2 ; 1=$ Holstein, 2= Jersey)

nlac $_{m}=$ efecto fijo de la $m$-ésima lactancia ( $m=$ $1-5 ; 1=1,2=2,3=3,4=4,5=\geq 5$ lactancias)

eco $_{n}=$ efecto fijo de la $n$-ésima ecozona $(n=$ 1 - 7)

$e_{i j k l m n}=$ efecto aleatorio residual USDA es:

El modelo estadístico para vida productiva

$$
Y_{i j k l}=\mu o+e n d o_{i}+h a n_{j}+r a z a_{k}+e c o l+e_{i j k l}
$$

Donde:

$Y_{i j k l}=$ vida productiva USDA (meses)

$\mu O=$ media general de la variable de respuesta endo $o_{i}=$ efecto fijo de la $i$-ésima endogamia $(i=$ $0 \ldots$; porcentaje de endogamia)

$h a n_{j}=$ efecto aleatorio del j-ésimo año de nacimiento

$r a z a_{k}=$ efecto fijo de $k$-ésima raza $(k=1-2 ; 1=$ Holstein, 2= Jersey)

eco $o_{l}=$ efecto fijo de la $l$-ésima ecozona $(l=1-7$

$e_{i j k l}=$ efecto aleatorio residual
La tercera fase fue la estimación del riesgo (Razón de posibilidades) de que una vaca con niveles altos de endogamia $(\geq 6,25 \%)$ (Romero et ál. 2006), presenten parámetros productivos bajos según la raza y el número de lactancia. Esto se realizó mediante el procedimiento PROC LOGISTIC, SAS/STAT® ver 9.2 .

\section{RESULTADOS Y DISCUSIÓN}

\section{Estadística descriptiva}

En total hubo un $17,9 \%(n=47$ 221) de animales con algún grado de endogamia en todas las razas, valor un poco mayor al reportado por Hudson y Van Vleck (1984) de 16,89\%. Por el contrario el estudio de Romero et ál. (2006) evidenció un 6,04\% de individuos endogámicos en vacas lecheras de Costa Rica. Esta diferencia podría deberse a que la población de fincas seleccionada por Romero et ál. fue más baja, así como un mayor porcentaje de animales Jersey en nuestro estudio. Adicionalmente, el programa VAMPP Bovino no contaba, en 1996, cuando Romero et ál. Hicieron su estudio, con una base de datos de toros tan completa como la actual, ni contaba con el motor de búsqueda de ancestros comunes para la estimación del coeficiente de endogamia que ahora posee. Por otra parte, a nivel mundial, se ha observado un incremento gradual de la endogamia en los hatos lecheros, y que también ha ocurrido en Costa Rica; así por ejemplo, en los Estados Unidos de América, la tasa de variación interanual de $\mathrm{F}$ fue de 0,14 y 0,24 , para Holstein y Jersey, para el período comprendido entre 1994 y 2004 (AIPL 2005, USDA 
2012). Mientras, como se observa en la Figura 1, en Costa Rica hubo una tendencia creciente y similar en los niveles de endogamia de la raza Holstein y Jersey en el período de 1995 a 2008. En general, el comportamiento observado es no lineal, de la misma forma que lo reporta Sewalem et ál. (2006) en un estudio hecho en Estados Unidos de América.

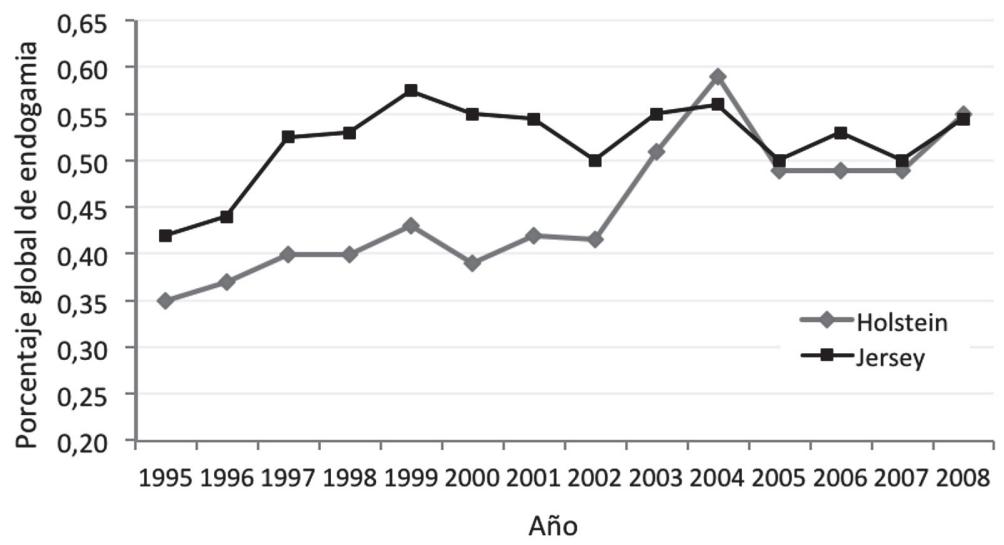

Fig. 1. Tendencias de consanguinidad para las razas Holstein y Jersey según el año de nacimiento, en fincas lecheras de Costa Rica, período 1995-2010.

En relación con la variable producción corregida a 305 d se encontró un promedio general de 5672,3 kg $(n=241399)$ y de vida productiva según el cálculo USDA (VPU) de 28,8 m ( $n=91$ 162), estos valores están dentro de los parámetros normales de vacas en producción lechera.

\section{Niveles de endogamia}

La raza Holstein presentó 22,6\% de vacas con algún grado de endogamia, mientras que en la raza Jersey fue un 20,3\%. Estos son similares a los reportados por Vargas y Gamboa (2008) con $21,2 \%$ para Holstein y $23,2 \%$ para Jersey, con la diferencia de que en nuestro el mayor porcentaje lo tiene Holstein.

El promedio general fue de $\mathrm{F}=0,42 \%$, con valores individuales que oscilan entre cero y $46,88 \%$. Esto confirma lo reportado por Casas y Tewolde (2001), de $0,44 \%$ en vacas lecheras de Costa Rica. Por otra parte, el promedio general de endogamia en los animales con $\mathrm{F}>0$ es de $\mathrm{F}=2,36 \%$.

El promedio general de consanguinidad (incluyendo los animales con un aparente $\mathrm{F}=0$ ) para la Holstein y Jersey fue de $0,49 \%$ y $0,55 \%$, respectivamente. Estos valores son más bajos que los encontrados en Vargas y Gamboa (2008), de $0,75 \%$ y $1,25 \%$ para las mismas razas en Costa Rica. En ambos estudios, el grupo racial Jersey tiene mayor media del coeficiente de endogamia que la Holstein (Cuadro 3). Si no se consideran los animales con aparentes $\mathrm{F}=0$, al hacer el cálculo de la media de $\mathrm{F}$, la raza que presenta un mayor promedio es la Jersey con 2,68\%, seguida de la Holstein con 2,16\% (Cuadro 4). Esto podría deberse, posiblemente, a que la población de vacas Jersey es más pequeña y, por ello, hay menor cantidad disponible de toros de raza Jersey destinados a inseminación artificial lo que predispone a que ocurran mayor cantidad de cruces endogámicos en esta raza.

La raza Jersey fue la que tuvo mayor porcentaje de vacas con grados de endogamia considerados de riesgo para obtener menor rendimiento productivo. Estos niveles de riesgo son $\mathrm{F} \geq 5 \% \mathrm{y} \geq 6,25 \%$ (Florio 2005) que son los valores máximos permisibles de consanguinidad en ganado bovino (Cuadro 3). 
Cuadro 3. Resumen estadístico de endogamia en los animales en estudio y sus diferentes niveles de riesgos, en fincas lecheras de Costa Rica, período 1995-2010.

\begin{tabular}{lccc}
\hline & & Raza & Jersey \\
\hline $\mathrm{N}$ & & Holstein & 87072 \\
Media del \% Consanguinidad & & 121076 & 0,55 \\
Mínimo (\%) & & 0,49 & 0,0 \\
Máximo (\%) & & 0,0 & 37,50 \\
& & 46,88 & $37,4(36,7-38,1)$ \\
Endogamia a diferentes & $\geq 2,5 \%$ & $22,0(21,6-22,5)$ & $10,6(10,1-11,0)$ \\
niveles de riesgo (IC 95\%) & $\geq 5,0 \%$ & $5,1(4,9-5,4)$ & $7,8(7,4-8,2)$ \\
\hline
\end{tabular}

*Toma en cuenta los animales sin endogamia.

Cuadro 4. Promedios de indicadores productivos de vacas de la raza Holstein, Jersey con algún grado de consanguinidad, en fincas lecheras de Costa Rica, período 1995-2010.

\begin{tabular}{lrrrr}
\hline Variable & \multicolumn{2}{c}{ Holstein } & \multicolumn{2}{c}{ Jersey } \\
\cline { 2 - 5 } & $\mathrm{N}$ & Media & $\mathrm{N}$ & Media \\
\hline Endogamia (\%) & 27365 & 2,16 & 17702 & 2,68 \\
Producción corregida 305d (kg) & 30635 & 7534 & 22543 & 5667 \\
Vida productiva USDA (meses) & 11405 & 27,89 & 7559 & 31,60 \\
\hline
\end{tabular}

*Toma en cuenta solo los animales endogámicos.

Por la alta tasa de uso de semen de origen en Estados Unidos de América, y por el uso del BLUP (Mejor Predictor Lineal Insesgado por sus siglas en inglés) como método de estimación del valor genético de sus sementales (Henderson 1988), con fines de selección, es probable que esa sea, en parte, una de las razones para el incremento observado en los índices de endogamia en los hatos nacionales, tal como se ha reportado en otras latitudes (Zhang et ál. 2003, Pedersen et ál. 2009, Kasarda y Kadlečík 2011); sin embargo, para efectos de este estudio tal efecto no se determinó.

\section{Curvas de vida productiva}

En la Figura 2 se puede observar la distribución de Weibull que muestra diferencias entre las medianas de vida productiva, según la fórmula de la USDA, de las razas Holstein y Jersey a niveles de endogamia altos $(\mathrm{F} \geq 6,25 \%)$, siendo de $29,5 \mathrm{~m}$ y $41,4 \mathrm{~m}$ respectivamente; mientras, en los animales con niveles de endogamia bajos ( $\mathrm{F}<6,25 \%$ ), fueron de $19,2 \mathrm{~m}$ y $22,1 \mathrm{~m}$. En general, la tendencia indica que hay mayor vida productiva en vacas con niveles bajos de endogamia. Así, la diferencia más notoria es de $+19,3$ m de VPU en raza Jersey para animales con $\mathrm{F}<6,25 \%$ respecto a niveles altos.

Es muy importante considerar este aspecto debido a que pueden ocurrir pérdidas económicas importantes en una lechería a través de la disminución de producción láctea cuando tiene animales con alta endogamia, principalmente cuando el grupo racial es Jersey. 

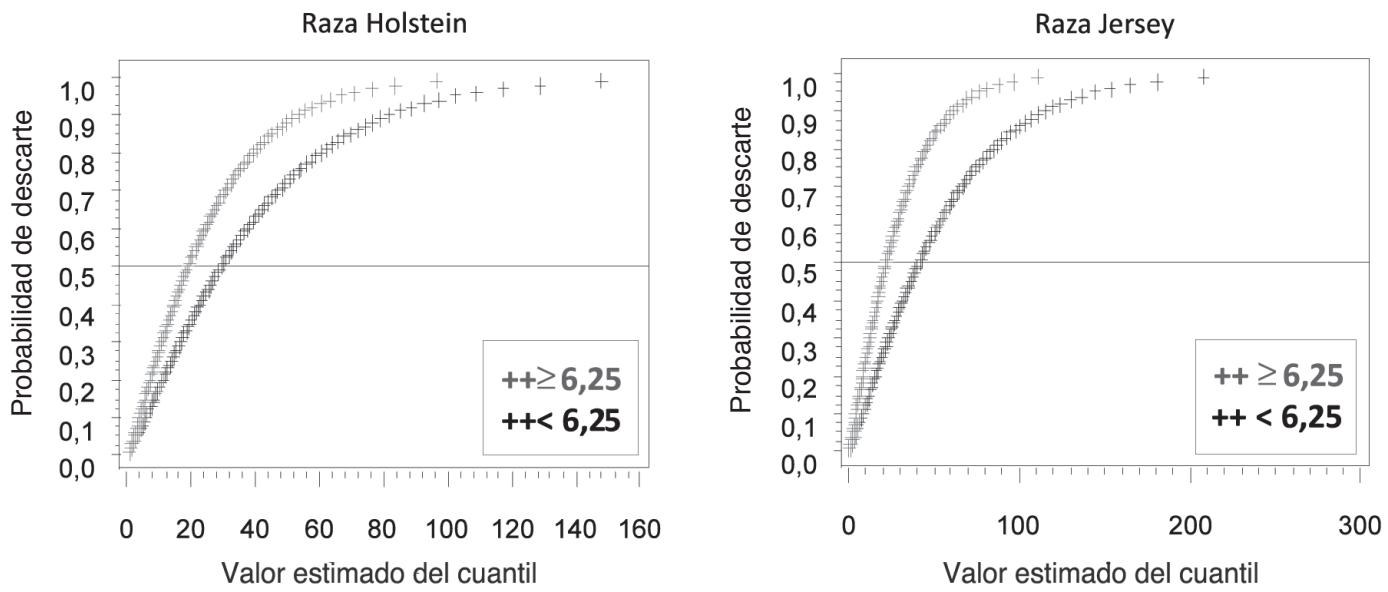

Fig. 2. Comparación de vida productiva (meses) según el cálculo USDA en Holstein y Jersey entre las que tienen un coeficiente de endogamia $\geq 6,25 \%$ y $<6,25 \%$, en fincas lecheras de Costa Rica, período 1995-2010.

Otros estudios plantean que las pérdidas de producción por depresión endogámica se dan en los animales jóvenes; es decir, en las primeras lactancias en donde existe una mayor producción, esto justifica las mayores pérdidas financieras que se dan por F alta (Thompson et ál. 2000).

\section{Efecto absoluto de la endogamia sobre la producción láctea y la vida productiva}

Se detectó $+140,4 \mathrm{~kg}$ de producción de leche (PC305d) en las vacas con $\mathrm{F}<6,25 \%$ $(\mathrm{p}<0,01)$, dato que concuerda con estudios de Cassell (1999) y Smith et ál. (1998), quienes reportan $-37 \mathrm{~kg}$ y $-177 \mathrm{~kg}$ por cada $1 \%$ de consanguinidad. Esto demuestra un efecto negativo significativo de la endogamia sobre producción de leche en los hatos lecheros de Costa Rica. Este efecto negativo, sin embargo, no es similar en todos los estudios; así, Pacheco et ál. 2010, no encontraron diferencias significativas en la producción láctea de vacas Holstein en las zonas áridas de México.

Asimismo, hubo efecto de todas las ecozonas y del número de lactancias sobre los PC305d $(\mathrm{p}<0,001)$. De esto, es importante destacar que en la zona ecológica bh-T (Bosque Húmedo Tropical) se producen $-1921,9$ PC305d $(p<0,001)$ que en el bp-MB (Bosque Pluvial Montano Bajo) y que en la primera lactancia hay menor producción que en las demás. También, se puede observar que hubo mayor producción $(+22 \mathrm{~kg}$ ) cuando la época de parto fue verano $(\mathrm{p}<0,05)$ Esto señala la importancia de considerar otros factores o variables en el modelo que pueden incidir en la variable dependiente además del nivel de consanguinidad (Cuadro 5). 
Cuadro 5. Efecto de la endogamia $\geq 6,25 \%$, época de parto, razas, ecozona y lactancias sobre Producción corregida a 305 d., en fincas lecheras de Costa Rica, período 1995-2010.

\begin{tabular}{|c|c|c|c|c|}
\hline Variable & Estrato & Estimado absoluto & Error estándar & $\mathrm{P}$ \\
\hline Intercepto & 0 & 5838,37 & 81,95 & $<, 0001$ \\
\hline \multirow[t]{2}{*}{ Endogamia } & $\geq 6,25 \%$ & 0 & . & . \\
\hline & $<6,25 \%$ & 140,43 & 24,62 & $<, 0001$ \\
\hline \multirow[t]{2}{*}{ Razas } & Jersey & 0 & . & . \\
\hline & Holstein & 1786,30 & 22,76 & $<, 0001$ \\
\hline \multirow[t]{7}{*}{ Ecozonas } & $\mathrm{Bp}-\mathrm{mb}$ & 0 & . & . \\
\hline & Bh-mb & $-297,64$ & 102,49 & 0,0037 \\
\hline & Bh-p & $-433,96$ & 97,75 & $<, 0001$ \\
\hline & Bh-t & $-1921,88$ & 205,05 & $<, 0001$ \\
\hline & Bmh-mb & $-842,40$ & 106,61 & $<, 0001$ \\
\hline & Bmh-p & $-554,53$ & 94,37 & $<, 0001$ \\
\hline & Bmh-t & $-1139,39$ & 92,18 & $<, 0001$ \\
\hline \multirow[t]{5}{*}{ Lactancias } & $\geq 5^{\mathrm{a}}$ & 0 & . & . \\
\hline & $1^{\mathrm{a}}$ & $-368,10$ & 18,23 & $<, 0001$ \\
\hline & $2^{\mathrm{a}}$ & $-197,55$ & 18,93 & $<, 0001$ \\
\hline & $3^{\mathrm{a}}$ & $-98,64$ & 20,13 & $<, 0001$ \\
\hline & $4^{\mathrm{a}}$ & $-61,40$ & 22,04 & 0,0053 \\
\hline \multirow[t]{2}{*}{ Época de parto } & Lluviosa & 0 & . & . \\
\hline & Seca & 21,98 & 11,31 & 0,0519 \\
\hline
\end{tabular}

bp-MB= bosque pluvial montano bajo; bh- $\mathrm{MB}=$ bosque húmedo montano bajo; bh- $\mathrm{P}=$ bosque húmedo premontano; bh- $\mathrm{T}=$ bosque húmedo tropical; bmh-MB= bosque muy húmedo montano bajo; bmh- $\mathrm{P}=$ bosque muy húmedo premontano; bmh-T= bosque muy húmedo tropical.

\section{Riesgo de efectos negativos de endogamia $\geq 6,25 \%$ sobre parámetros productivos}

Los animales con endogamia alta tienen 1,31 veces el riesgo (IC 95\%: 1,2-1,4) de PC305d $<5000 \mathrm{~kg} \mathrm{y}<6500$ que los animales con $\mathrm{F}$ bajo, para las Jersey y Holstein, respectivamente (Cuadro 6). Este resultado se complementa con el de modelos mixtos; lo que indica, nuevamente, que el impacto negativo que tiene la endogamia $\geq 6,25 \%$ es consistente sobre la PC $305 \mathrm{~d}$.

La zona ecológica también tiene influencia sobre PC305d $(p<0,05)$. Las vacas que están en la ecozona bosque húmedo tropical (bh-T) tienen 23,05 veces el riesgo de producir menos leche $(\mathrm{kg} / 305 \mathrm{~d})$ que las del bosque pluvial montano bajo (bp-MB). Asimismo, las vacas de la primera lactancia tienen 1,53 veces la probabilidad de producir menos leche en la primera lactancia en relación a la tercera (IC: 1,45-1,62). Por otra parte, los animales que parieron en época lluviosa tuvieron 1,08 (IC95\%: 1,04-1,12) veces el riesgo de tener bajas producciones, comparado con los animales que parieron en época seca $(\mathrm{p}<0,05)$ (Cuadro 6). 
Cuadro 6. Razón de posibilidades de las diferentes variables en estudio con respecto a producción corregida a 305 d., en fincas lecheras de Costa Rica, período 1995-2010.

\begin{tabular}{|c|c|c|c|c|}
\hline \multirow{2}{*}{$\begin{array}{l}\text { Variable } \\
\text { Endogamia }\end{array}$} & \multirow{2}{*}{$\begin{array}{l}\text { Efecto } \\
\geq 6,25 \% \text { vs. }<6,25 \%\end{array}$} & \multirow{2}{*}{$\begin{array}{c}\text { Razón de posibilidades } \\
1,31\end{array}$} & \multicolumn{2}{|c|}{ Intervalos de confianza (95\%) } \\
\hline & & & 1,22 & 1,42 \\
\hline Razas & Holstein vs. Jersey & 0,88 & 0,85 & 0,92 \\
\hline \multirow[t]{6}{*}{ Ecozonas } & Bh-mb vs. Bp-mb & 1,90 & 1,77 & 2,04 \\
\hline & Bh-p vs. Bp-mb & 1,73 & 1,62 & 1,85 \\
\hline & Bh-t vs. Bp-mb & 23,05 & 17,71 & 30,00 \\
\hline & Bmh-mb vs. Bp-mb & 2,60 & 2,40 & 2,81 \\
\hline & Bmh-p vs. Bp-mb & 2,23 & 2,08 & 2,38 \\
\hline & Bmh-t vs. Bp-mb & 4,06 & 3,79 & 4,35 \\
\hline \multirow[t]{4}{*}{ Lactancias } & $1^{\mathrm{a}}$ vs. $3^{\mathrm{a}}$ & 1,53 & 1,45 & 1,62 \\
\hline & $2^{\mathrm{a}}$ vs. $3^{\mathrm{a}}$ & 1,18 & 1,11 & 1,25 \\
\hline & $4^{\mathrm{a}}$ vs. $3^{\mathrm{a}}$ & 0,96 & 0,89 & 1,03 \\
\hline & $\geq 5^{\mathrm{a}}$ vs. $3^{\mathrm{a}}$ & 0,88 & 0,82 & 0,94 \\
\hline Época de parto & Lluviosa vs., seca & 1,08 & 1,04 & 1,12 \\
\hline
\end{tabular}

bp-MB= bosque pluvial montano bajo; bh- $\mathrm{MB}=$ bosque húmedo montano bajo; bh- $\mathrm{P}=$ bosque húmedo premontano; bh- $\mathrm{T}=$ bosque húmedo tropical; bmh-MB= bosque muy húmedo montano bajo; bmh- $\mathrm{P}=$ bosque muy húmedo premontano; bmh-T= bosque muy húmedo tropical.

Por otro lado, no hubo un efecto significativo de la endogamia sobre los animales la vida productiva de los animales $(\mathrm{OR}=1,08$; IC 95\%: 0,94-1,24). Sewalem et ál. (2006) realizó un estudio similar en Canadá, pero utilizaron un rango de $\mathrm{F}$ entre $\geq 6,25 \%$ y $<12,5 \%$, con riesgo incrementado de descarte prematuro en ambas razas, con valores de OR de 1,19 en Holstein y 1,14 Jersey $(p<0,05)$. Nuestros resultados son muy cercanos, pero con el alto número de animales estudiados, consideramos que la ausencia de significancia estadística en la diferencia de riesgo es de altísima validez (Cuadro 7).

Las zonas ecológicas también tienen riesgo significativo sobre la vida productiva; así los animales que viven en la ecozona del bosque húmedo tropical (bh-T) tienen 6,24 veces el riesgo de tener una vida productiva (USDA) baja, respecto a los animales que habitan en bosque pluvial montano bajo (bp-MB). Asimismo, los animales sobre 4 lactancias tienen menor probabilidad de tener bajas producciones respecto a las de la $3^{\circ}$ (Cuadro 7). 
Cuadro 7. Razón de posibilidades de las diferentes variables en estudio respecto a vida productiva según USDA, en fincas lecheras de Costa Rica, período 1995-2010.

\begin{tabular}{|c|c|c|c|c|}
\hline \multirow{2}{*}{$\begin{array}{l}\text { Variable } \\
\text { Endogamia }\end{array}$} & \multirow{2}{*}{$\begin{array}{l}\text { Efecto } \\
\geq 6,25 \% \text { vs. }<6,25 \%\end{array}$} & \multirow{2}{*}{$\frac{\text { Razón de posibilidades }}{1,08}$} & \multicolumn{2}{|c|}{ Intervalos de confianza (95\%) } \\
\hline & & & 0,94 & 1,24 \\
\hline Razas & Holstein vs. Jersey & 1,34 & 1,25 & 1,44 \\
\hline \multirow[t]{6}{*}{ Ecozona } & Bh-mb vs. Bp-mb & 1,50 & 1,34 & 1,69 \\
\hline & Bh-p vs. Bp-mb & 1,52 & 1,37 & 1,69 \\
\hline & Bh-t vs. Bp-mb & 6,24 & 3,49 & 11,15 \\
\hline & Bmh-mb vs. Bp-mb & 1,72 & 1,50 & 1,98 \\
\hline & Bmh-p vs. Bp-mb & 1,93 & 1,74 & 2,15 \\
\hline & Bmh-t vs. Bp-mb & 1,96 & 1,74 & 2,20 \\
\hline \multirow[t]{4}{*}{ Lactancias } & $1^{\mathrm{a}}$ vs. $3^{\mathrm{a}}$ & 5,91 & 2,53 & 13,80 \\
\hline & $2^{\mathrm{a}}$ vs. $3^{\mathrm{a}}$ & 3,90 & 1,53 & 9,92 \\
\hline & $4^{\mathrm{a}}$ vs. $3^{\mathrm{a}}$ & 0,93 & 0,25 & 3,51 \\
\hline & $\geq 5^{\mathrm{a}}$ vs. $3^{\mathrm{a}}$ & 0,84 & 0,14 & 5,06 \\
\hline
\end{tabular}

bp- $\mathrm{MB}=$ bosque pluvial montano bajo; bh- $\mathrm{MB}=$ bosque húmedo montano bajo; bh- $\mathrm{P}=$ bosque húmedo premontano; bh- $\mathrm{T}=$ bosque húmedo tropical; bmh- $\mathrm{MB}=$ bosque muy húmedo montano bajo; bmh- $\mathrm{P}=$ bosque muy húmedo premontano; bmh-T= bosque muy húmedo tropical.

\section{CONCLUSIONES}

La endogamia en los hatos lecheros de Costa Rica ha ido en aumento con los años, produciendo un porcentaje importante de animales endogámicos y con niveles de riesgo, tanto en la raza Holstein como en la Jersey, en la que se observa el mayor porcentaje. El efecto neto de la endogamia fue negativo sobre la mediana de vida productiva medida según el método USDA, siendo $-10 \mathrm{~m}$ y $-19 \mathrm{~m}$ para las vacas Holstein y Jersey con coeficiente de endogamia alta. Asimismo, los animales con $\mathrm{F}<6,25 \%$ presentaron mayor producción de leche corregida a $305 \mathrm{~d}$.

Con los resultados encontrados en este estudio se demuestra que existe un efecto negativo y significativo sobre la producción corregida a $305 \mathrm{~d}$ y la vida productiva (USDA) en los animales con $\mathrm{F}>6,25$ en vacas Holstein y Jersey de hatos lecheros especializados de Costa Rica.

\section{LITERATURA CITADA}

AIPL (Animal Improvement Programs Laboratory). 2005. Subject: Inbreeding trends. Consultado: 04 de septiembre de 2011. Disponible: http://aipl.arsusda. gov/dynamic/inbrd/current/kindx.html

CARAVIELLO D. 2004. Duración de la vida productiva. Reproducción y Genética. No ${ }^{\circ}$. 612. Consultado: 02 de septiembre de 2011. Disponible en: http:/ babcock.wisc.edu/sites/default/files/documents/ productdownload/du_612.es_.pdf

CASAS E., TEWOLDE A. 2001. Evaluación de características relacionadas con la eficiencia reproductiva de genotipos criollos lecheros en el trópico húmedo. Archivo Latinoamericano de Producción Animal. 9(2):63-67.

CASSELL B.G. 1999. Effect of Inbreeding on Lifetime Performance of Dairy Cows. Dept of Dairy Science, Virginia Polytechnic Institute and State Univ, Blacksburg, VA. Advances in Dairy Technology. 11:13-23. Consultado: 10 de setiembre de 2011. Disponible en: http://www.wcds.ca/proc/1999/ Manuscripts/Chapt\%2002-\%20Cassell.pdf

CHIRINOS Z., GONZÁLEZ C., MADRID N., RIVERA J. 1999. Vida útil, longevidad y causas de eliminación 
en vacas mestizas doble propósito. Revista científica, FCV-LUZ. 9(6):477-484.

COLINA J., VERDE O., HAHN M., BARRIOS D. 2000. Comportamiento productivo de un rebaño Holstein puro bajo condiciones tropicales. Rev. Fac. Cs. Vets. 41(1-3):25-32.

DE ALBA J. 1964. Reproducción y genética animal. Turrialba: SIC. $446 \mathrm{p}$.

DUCROCQ V., SOLKNER J. 1998. Implementation of a routine breeding value evaluation for longevity of dairy cows using survival analysis techniques. Dept. of Livestock. Sci. 33. Vienna, Austria. Consultado 02 de setiembre de 2011. Disponible en: http://wwwsgqa.jouy.inra.fr/IMG/pdf/armil.pdf

FLORIO J. 2005. Consanguinidad en la ganadería bovina. en Manual de Ganadería Doble propósito: GonzálezStagnaro C. y Soto Belloso E. ed. Fundación GIRARZ. (pp 129 - 134) 701 p. Consultado 1 de setiembre de 2011. Disponible en: http://www.avpa. ula.ve/libros_online.html

HENDERSON C.R. 1988. Theoretical basis and computational methods for a number of different animal models. Proceedings of the animal model workshop, Edmonton, Alberta, Canada. J. Dairy Sci. 71(supp.2):1-16.

HOLDRIDGE L. 1987. Ecología basada en zonas de vida. IICA: San José. 219 p.

HUDSON G.F.S., VAN VLECK L.D. 1984. Inbreeding of artificially bred dairy cattle in the northeastern United States. J. Dairy Sci. 67(1):161-170.

KASARDA R., KADLEČÍK O. 2011. Influence of Maximum Inbreeding Avoidance under BLUP EBV Selection on Pinzgau Population Diversity. Animal Science and Biotechnologies 44(1):255-259.

NOORDHUIZEN J.P.T.M., BUURMAN J. 1984. Veterinary automated management and production control programme for dairy farms (VAMPP), the application of MUMPS for data processing. Veterinary Quarterly 6:62-77.

McPARLAND S., KEARNEY F., RATH M., BERRY D. 2007. Inbreeding Effects on Milk Production, Calving Performance, Fertility, and Conformation in Irish Holstein-Friesians. 90(9):4411-4419.

MAIWASHE A., NEPHAWE K., THERON H. 2008. Estimates of genetic parameters and effect of inbreeding on milk yield and composition in South African Jersey cows. South African Journal of Animal Sci. 38(2):119-125.

ORREGO J., DELGADO A., ECHEVARRÍA L. 2003. Vida productiva y principales causas de descarte de Vacas Holstein en la Cuenca de Lima. Rev. Investig. Vet. Perú. 14(1):68-73.

PACHECO P., HERNANDEZ J.R., MAGANA F. 2010. Efecto de la consanguinidad sobre la producción láctea en vacas Holstein en el establo "La Estrella",
León, Gto., México. Revista Chapingo Serie Zonas Áridas. 9:71-75.

PEDERSEN L.D., SØRENSEN A.C., BERG P. 2009. Marker-assisted selection can reduce true as well as pedigree-estimated inbreeding. J. Dairy Sci. 92(5):2214-2223.

ROMERO J.J. 2005. Appraisal of the epidemiology of Neospora caninum infection in Costa Rican dairy cattle. Ph.D. Thesis. Wageningen University. The Netherlands. 137 p.

ROMERO J.J., ROJAS J., PÉREZ E. 2006. Relación de la endogamia con la retención de membranas fetales en vacas de hatos lecheros especializados de Costa Rica. Ciencias Veterinarias. 24(1):79-89.

SAS Inst. Inc. 2009. SAS/STAT® User's Guide: Statistics; Version 9.2. $2^{\text {nd }}$ ed. Consultado: 25 de agosto de 2011. Disponible en: http://support.sas.com/ documentation/cdl/en/statug/63033/HTML/default/ viewer.htm\#titlepage.htm

SEWALEM A., KISTEMAKER G.J., MIGLIOR F., VAN DOORMAAL B.J. 2006. Analysis of inbreeding and its relationship with functional longevity in Canadian dairy cattle. J. Dairy Sci. 89(6):2210-2216.

SMITH L.A., CASSELL B.G., PEARSON R.E. 1998. The Effects of Inbreeding on the Lifetime Performance of Dairy Cattle. J. Dairy Sci. 81(10):2729-2737.

THOMPSON J.R., EVERETT L.W., HAMMERSCHMIDT N.L. 2000. Effects of Inbreeding on Production and Survival in Holsteins. J. Dairy Sci. 83(8):1856-1863.

USDA (United States Department of Agriculture). 2012. Trend in Inbreeding Coefficients for Holstein or Red \& White. Consultado: 11 de febrero de 2013. Disponible en: http://aipl.arsusda.gov/eval/summary/ inbrd.cfm

VAN RADEN P.M., DEMATAWEWA C.M., PEARSON R.E., TOOKER M.E. 2006. Productive Life Including All Lactations and Longer Lactations with Diminishing Credits. J. Dairy Sci. 89(8):3213-3220.

VARGAS B., GAMBOA G. 2008. Estimación de tendencias genéticas e interacción genotipo $\mathrm{x}$ ambiente en ganado lechero de Costa Rica. Téc. Pecu. Méx. 46(4):371-386.

VARGAS B., ULLOA J. 2008. Relación entre curvas de crecimiento y parámetros reproductivos en grupos raciales lecheros de distintas zonas agroecológicas de Costa Rica. (en línea). Livestock Research for Rural Development. 20 (103). Consultado: 04 de septiembre de 2011. Disponible en: http://www.lrrd. org/lrrd20/7/varg20103.htm

WEIGEL K., LIN S. 2000. Use of Computerized Mate Selection Programs to Control Inbreeding of Holstein and Jersey Cattle in the Next Generation. J Dairy Sci. 83(4):822-828.

WIGGANS G.R., VANRADEN P.M., ZUURBIER J. 1995. Calculation and use of inbreeding coefficients for 
genetic evaluation of United States dairy cattle. J. Dairy Sci. 78(7):1584-1590.

WRIGHT S. 1922. Coefficients of inbreeding and relationship. American Naturalist 56:330-338.
ZHANG H., ZHANG Y., ZHANG Q. 2003. Pre-selecting young bulls before progeny-testing with markerassisted BLUP. Chinese Science Bulletin. 48(3):259265.

(c) $\$ \bigoplus$ Todos los derechos reservados. Universidad de Costa Rica. Este artículo se encuentra licenciado con Creative Commons Reconocimiento-NoComercial-SinObraDerivada 3.0 Costa Rica. Para mayor información escribir a rac.cia@ucr.ac.cr 